\title{
La construcción de conocimiento profesional e identidad de directoras de establecimientos educacionales chilenos
}

\author{
Construction of Professional Knowledge and Identity \\ by Female Principals of Chilean Educational \\ Establishments
}

\section{Andrea Elizabeth Carrasco Sáez}

Universidad de Chile

\section{Resumen}

\begin{abstract}
Las reflexiones de este artículo son resultado de una investigación realizada a partir de los discursos de un grupo de directoras de establecimientos educacionales de enseñanza básica de Chile sobre el proceso de construcción de conocimiento profesional e identidades directivas. Esta investigación fue de carácter cualitativo, y se posicionó desde un enfoque biográfico narrativo. En ella, participaron diez directoras de escuelas municipales y particulares subvencionadas. Los resultados seńalan que la construcción de conocimiento profesional e identidad es un proceso situado, de interacción social, en el que se conjugan saberes teóricos, prácticos y reflexivos, todos a la base de la experiencia personal y profesional de las directoras y con su entorno cotidiano.
\end{abstract}

Palabras claves: conocimiento profesional, identidad, interacción social, directoras.

(c) $2021 \mathrm{PEL}, \mathrm{http}: / / \mathrm{www}$.pensamientoeducativo.org - http://www.pel.cl 


\begin{abstract}
The reflections in this paper are the result of a study based on the discourses of a group of female principals of elementary education establishments in Chile regarding the process of constructing professional knowledge and managerial identities. The research is qualitative and is approached from a biographical-narrative perspective. Ten female principals of municipal and subsidized private schools took part. The results indicate that the construction of professional knowledge and identity is a situated process, involving social interaction, which combines theoretical, practical, and reflective knowledge, all based on the personal and professional experience of the principals and with their daily environment.
\end{abstract}

Keywords: professional knowledge, identity, social interaction, directors.

\title{
Introducción
}

Diversos estudios nacionales e internacionales han demostrado que el desempeño y liderazgo del director/a es de gran influencia e importancia para la mejora y eficacia de la institución educativa, como un factor estratégico (Leithwood, Day, Sammons, Harris, \& Hopkins, 2006; Weinstein y Muñoz, 2012; Bolívar, 2019; Maureira y Garay, 2019). Los estudios han develado que el conocimiento, las fuertes identidades directivas (Bolívar y Ritacco, 2019) y el liderazgo de las y los directores influyen directamente en el fortalecimiento de los aprendizaje de las y los estudiantes (Louis, Leithwood, Wahlstrom, \& Anderson, 2010) y en el aprendizaje de la comunidad educativa en su conjunto (Barber y Mourshed, 2008; Leithwood, 2009), lo que ayuda a promover la autonomía escolar (Bolívar, 2019). Por lo tanto, es relevante indagar y analizar cómo aprenden, cómo construyen el conocimiento profesional y cómo son las identidades de las y los directores del país, para fortalecer el desarrollo profesional directivo y su selección y evaluación.

A raíz de lo anterior, durante los últimos años, la política pública en educación se ha focalizado en impulsar un conjunto de acciones que han permitido definir las funciones, atribuciones y ámbitos de acción de las y los directores escolares del país, además de promover su selección al cargo y su formación continua. A saber, ha habido un foco en

(...) Mejorar las capacidades de liderazgo disponible en el sistema, ya sea asegurando el ingreso al cargo directivo de los candidatos más idóneos (por ejemplo, haciendo más atractiva la carrera directiva o fortaleciendo los procesos de selección), o bien desarrollando habilidades (y prácticas) de liderazgo efectivo en los directivos en ejercicio, principalmente a través de programas de formación” (Mineduc, 2016, p .21)

En este contexto, se han implementado una serie de leyes y reformas que han modificado los roles y funciones de las y los directores, definiendo nuevas atribuciones para el cargo: Ley General de Educación (№ 20.370, 2009), Ley de Inclusión Escolar (No 20.845, 2015), Ley de Sistema de Desarrollo Profesional Docente (No 20.903, 2016), además de la Ley de Calidad y Equidad de la educación (No 20.501, 2011) y la Ley No 20.006 (2005), que determinan los mecanismos de selección y evaluación de los cargos directivos en establecimientos municipalizados del país.

En lo que respecta a la distribución por género en los cargos directivos, según información extraída del Centro de Estudios (Mineduc, 2019), en la actualidad el cargo de director/a está ocupado en un 64\% por mujeres y en un $36 \%$ por hombres, porcentaje alto que demuestra la importancia que ha tomado el rol femenino en cargos 
directivos. Junto con lo anterior, resulta pertinente señalar que en lo relativo a los aprendizajes o conocimientos de las y los directores, las investigaciones en el país se han focalizado en estudiar mayormente el desarrollo de competencias directivas (Muñoz y Marfán, 2011; Uribe \& Celis, 2012), mas no los conocimientos, siendo las primeras la base para la construcción de los conocimientos profesionales de los directores. Considerando lo anterior, resulta relevante indagar tanto en el conocimiento profesional como en la identidad de las directoras del país.

Por lo anterior, este artículo busca comprender cómo se construye el conocimiento profesional y la identidad de las directoras de colegios municipales y particulares subvencionados en Chile, queriendo ser un aporte para la reflexión sobre la construcción del conocimiento profesional y la configuración de identidades en la dirección escolar.

\section{Marco conceptual}

\section{Conocimiento profesional directivo}

El conocimiento profesional de los directores/as y su generación resulta ser un proceso intrínseco a la experiencia humana y complejo, en tanto son múltiples los saberes que están implicados en su construcción (Pech y Morejón, 2018). Para que el conocimiento se produzca, se requiere la posibilidad de constituir una experiencia, y en la medida en que esta no se lleve a cabo, habrá información, pero no un auténtico saber (Dubet, 2011; Contreras y Pérez de Lara, 2010). Es por ello que a la hora de pensar la construcción de conocimiento resulta imposible separar al sujeto del objeto, ya que este se produce como parte de nuestro ser, de nuestras experiencias significadas en lo cotidiano, en la acción del ser humano (Tadeu da Silva, 2001; Heller, 1977). Esto implicará superar la tradicional separación entre los conocimientos disciplinares, pedagógicos, y lo práctico (Vaillant, 2019).

En esta línea, el conocimiento no es nunca un elemento aislado, sino que para existir y para su proceso de construcción requiere de la interacción y la significación con el entorno del sujeto. A este respecto, Tardif (2013) señala que el conocimiento está siempre marcado por el contexto en el cual se mueve la profesión, por lo que en este caso tiende a estar cruzado por controversias sociales y políticas propias del contexto socioeducativo. Así, en la construcción de conocimiento, las directoras ponen en juego el mundo ideológico y el sentido común (Hargreaves y Fullan, 2014).

Cuando se habla de conocimiento profesional, podemos hacer referencia a este como conocimiento práctico o de la práctica (Pérez Gómez, 1988), como percepción (Heller, 1977), como saberes, experiencias (Tardif, 2004, 2013), y como saberes personales. Sin embargo, al adentrarnos en estas definiciones, podemos observar que en la base de todas ellas encontramos una relación entre distintos niveles de conocimiento (racional y experiencial) que interactúan entre sí, provocando la emergencia del conocimiento profesional directivo (Marcelo, 2009).

En esta línea, Porlán-Ariza, Rivero y Martín del Pozo (1997) plantean que el conocimiento profesional es el resultado de la yuxtaposición de saberes que se expresan en dos dimensiones: una epistemológica (racionalexperiencial) y una psicológica (explícito-tácito). De igual manera, Tamir (2005) plantea que, en primera instancia, resulta de suma relevancia la diferencia y la relación que existe entre el conocimiento teórico y el práctico. En este marco, define que el conocimiento profesional debe ser entendido como el cuerpo de habilidades necesarias para funcionar con éxito en una profesión en específico. Además, el conocimiento profesional es heterogéneo y ecléctico, es decir, requiere de distintos componentes para su construcción (Shulman, 2005).

Se entiende que el conocimiento profesional, más que solo saberes teóricos, requiere siempre de aquellos saberes que surgen de las vivencias y la reflexión de los actores, otorgándole valor al contexto en el que las directoras ejercen su rol (Tardif, 2013). 
El proceso mediante el cual se construye el conocimiento profesional es social. Por ello, la directora no definirá su propio conocimiento profesional, sino que esto se hará mediante la interacción con otros sujetos educativos (Lave \& Wenger, 1991).

Sin embargo, esta interacción requiere de una capacidad reflexiva que permita recrear e involucrar racionalmente las practicas sociales. A este respecto, la práctica reflexiva sigue siendo un gran aliado, ya que mediante este proceso de reflexión los sujetos toman notas de las acciones, incorporándolas a sus herramientas (Giddens, 1992). En este marco, la práctica reflexiva se entiende como un proceso intencionado y sistemático mediante el cual el sujeto busca el aprendizaje sobre sí mismo y de la relación con otros, dotándole de poder para generar nuevos conocimientos profesionales, para enfrentarse a diversos escenarios (Giddens, 1992). Es así que, por un lado, las directoras interactúan con otros actores en el marco de dicha construcción, pero también en aquel proceso confluyen diversos tipos de conocimiento. A este respecto, Schön (1992) señala que los actores desarrollan una práctica profesional reflexiva a través de la solución de problemas que encuentran en su cotidianeidad, vinculándola con las acciones para tomar decisiones y metodologías para la innovación.

Los procesos reflexivos son en torno a la acción; por ende, no son independientes, se complementan y derivan en que la acción se vuelve progresivamente más tácita, mecánica e inconsciente cuando existen una trayectoria y rutina asociadas a ella (Poggi, 2001). Los procesos reflexivos son importantes ya que permiten la producción y movilización de conocimiento profesional (Campos, 2016).

El proceso mediante el cual se construye el conocimiento profesional de las directoras suele estar marcado por distintos tipos de promoción al interior de los espacios educativos. Es decir, una promoción vertical hace referencia a la posibilidad que tienen los docentes de realizar cursos y construir sus habilidades avanzando en la escala jerárquica de cargos presentes en las instituciones escolares; otra promoción es la horizontal, en la que lo relevante resulta ser la realización satisfactoria de cursos de formación continua en un mismo rol, sin aspiraciones a nuevos cargos como motor (UNESCO, 2007).

Finalmente, es posible seńalar que cada experiencia representa un andamiaje particular de conocimientos: de la práctica, para la práctica, y en la práctica (Simkins, 2005), que responde a una trayectoria de experiencias cimentada en sus historias de vida, de las reflexiones cotidianas, de la interacción con sus pares y, por supuesto, de su mismo actuar como sujeto de director/a en su espacio escolar. De esta forma, las directoras construyen su conocimiento mediante la relación continua de tres componentes: teóricos, prácticos y de la experiencia reflexiva de sus actos.

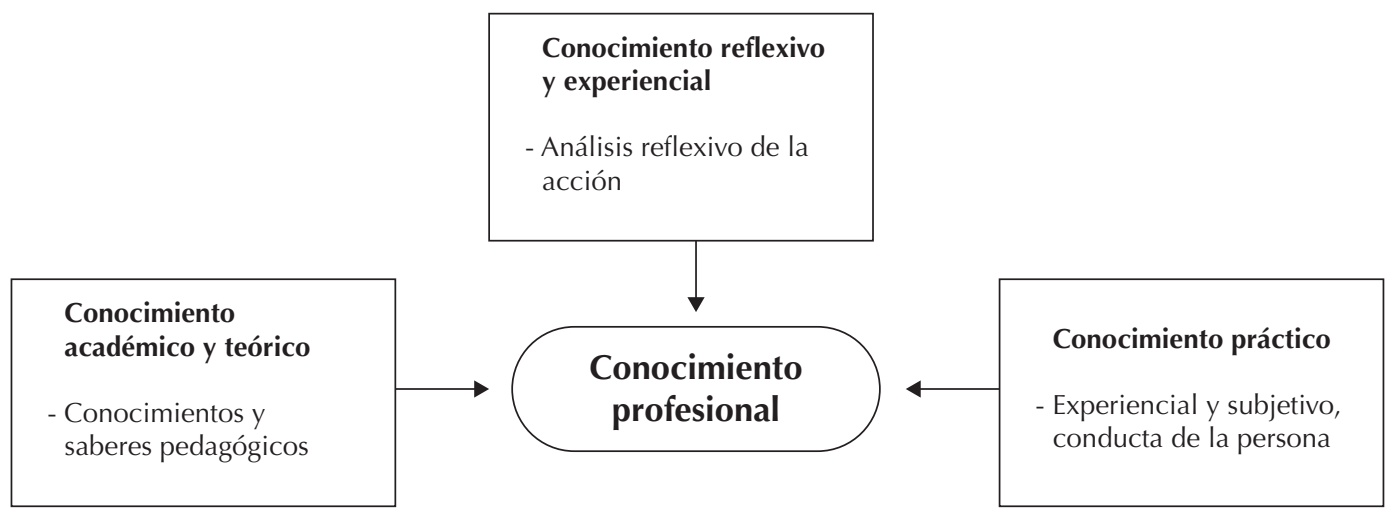

Figura 1. Sintesis de la construcción de conocimiento profesional de las directoras

Fuente: elaboración propia a partir de Demuth (2011), Poggi (2001), Porlán-Ariza et al. (1997), Shulman (2005), Tamir (2005), Tardif (2013). 


\section{Identidad y trayectoria de las directoras}

Hablar de identidad implica un proceso colectivo de autorreconocimiento, complementado con el reconocimiento de los demás y permeado por los diferentes cambios sociales que afectan directamente la configuración de la identidad (Vaillant, 2008).

En este contexto, para entender la identidad propia, se requiere pensar siempre en relación a la comunidad de pertenencia (Ricoeur, 1996; Ritacco y Bolívar, 2018). La identidad no niega en ningún caso el componente individual, sino que entiende que se está siempre en relación e interacción con otros (Wenger, 2001). La identidad no es algo que se posea; se desarrolla a lo largo de la vida, en una trayectoria, por lo que no es un atributo de una persona, sino que es un fenómeno relacional (Marcelo, 2010).

Es más, cuando hablamos de las directoras, un componente individual y personal relevante está asociado con las características propias del género, que influyen en cómo construyen su propia identidad, desde lo personal a lo colectivo profesional, y cómo se confrontan con las tensiones identitarias que las lleven a identificarse como profesoras-directoras (Vanegas \& Fuentealba, 2019). A este respecto, resulta imposible obviar que el rol de las directoras se establece en un espacio que tiende a ser masculinizado, en la medida en que detentan poder, y este es típicamente asociado a la figura masculina y de prácticas patriarcales (Barberá, Ramos, y Candela, 2011; Bustos, Oros, Álvarez, y Rebolledo, 2015; Armstrong \& Mitchell, 2017), espacio del cual las mujeres han sido históricamente excluidas. Así, no resulta extraño que la identidad de las directoras se vaya constituyendo en pugna con una visión que las pone constantemente en tensión a la hora de pensar sus identidades, conllevando muchas veces la elaboración de nuevas figuras que les permiten validarse frente a los demás, como la figura de la gerente ideal (Moorosi, 2007). Asumir responsabilidades adicionales a las de los hombres, vinculadas con el cuidado de la descendencia y el hogar, jornadas extensas y extras de trabajo, entre otros elementos (Cubillo \& Brown, 2003; Cabrera, 2005; Coronel, Llamas, Macías, \& Moreno, 2012; Gómez y Jiménez, 2019), generan complejidades que implican compatibilizar un trabajo de alta exigencia con la crianza de los hijos (Coleman, 2007). En algunos casos se genera hacer maternidad machista (Smithson \& Stokoe, 2005), escenario en el cual las mujeres toman su baja maternal por menor tiempo del concedido por ley. Todas estas acciones son evidencias de las formas de dominación en la que se ha insertado la construcción de identidad de las directoras, espacio que ha estado sometido a la producción de saberes desde una lógica heteronormativa, desigual y discriminatoria hacia las mujeres, por lo que se hace necesaria la transformación de estos espacios, así como impulsar el desarrollo de prácticas situadas en una pedagogía femenina (Troncoso, Follegati, y Stutzin, 2019). Por ello, el desafío sigue siendo superar las concepciones de que la administración en educación es una empresa masculina y que la racionalidad burocrática es una característica propia de la masculinidad hegemónica, las cuales reducen esta al accionar masculino y atentan contra el desarrollo de la práctica femenina en los cargos de liderazgo (Blackmore, 2017).

De todas formas, es menester entender que las identidades son sociales y que se desarrollan siempre en relación con las otras personas -o grupos sociales-, y son influenciadas por su contexto. Es decir, no son inamovibles, sino que más bien responden a un continuo movimiento en el cual los contextos históricos y culturales juegan un rol fundamental (Dubar, 2002; Crow \& Møller, 2017). Por lo tanto, no resulta extrańo que la identidad y las características del liderazgo en las mujeres directoras se den con relación a lo masculino (Jones, 2017).

Por ende, para adentrarnos en las identidades de las directoras resulta necesario indagar en sus elementos constitutivos enmarcados en una trayectoria que integra la dimensión personal y la profesional, revalorizando las creencias, sentimientos y motivaciones de la labor directiva (Bolívar, 2007), considerando el contexto o espacio social en el que se desarrollan. 
A este respecto, diversos estudios plantean que las identidades profesionales deben ser reconocidas para sí y para los otros, de tal manera que la aceptación del entorno se vuelve un elemento fundamental en su construcción. Desde esta lectura, el rol de las directoras depende de esta identidad profesional reconocida por otros, desligándose de la figura de autoridad impuesta; esto, porque se conforman al integrar puntos de vistas de otros acerca de las actuaciones personales, cuestión que otorga legitimidad a la labor (Ritacco y Bolívar, 2018).

En esta línea, y enfocado en el ejercicio educativo, Sammons y sus colegas (2007) plantean tres dimensiones que interactúan cuando se analizan las identidades profesionales: una identidad relacionada con la tradición de la enseńanza y las políticas educativas, una situada localmente y relacionada con la escuela y la institución o las instituciones por las que se ha transitado, y una personal, relativa a la vida fuera de la escuela. Por su parte, la formación inicial y continua influye en la construcción y reconstrucción de la identidad como un proceso transformativo (Carver, 2016). Así, la trayectoria juega un rol fundamental, ya que es en ella que se configura la identidad del director/a, junto con sus historias docentes, sus acontecimientos personales y experiencias profesionales, elementos que influyen en la construcción de conocimiento profesional. Al respecto, Morgade (2005) plantea que el devenir de un docente o un directivo/a es un proceso de subjetivación, y como proceso dura toda la vida, desde las primeras experiencias escolares de los docentes-directivos hasta la construcción de conocimiento profesional que se realiza en el trabajo en las escuelas.

En este sentido, resulta imperativo a la hora de analizar la construcción de conocimiento profesional de las directoras situarlas en sus propias trayectorias y contextos, para desde ahí lograr desarrollar un diálogo entre la heterogeneidad de saberes y conocimientos, donde estos deben inspirar a la acción, al compromiso social y al cuestionamiento de los procesos de normalización de relaciones de poder existentes (Cariño et al., 2017), sobre todo al interior de los establecimientos educativos.

\section{Marco metodológico}

Esta investigación, realizada durante el periodo 2018-2019, fue de carácter exploratoria, longitudinal y con producción de conocimiento a partir de fuentes primarias. El acercamiento al objeto de estudio se realizó mediante una metodología cualitativa, más específicamente desde la relación de dos enfoques particulares: comprensivointerpretativo (Berger y Luckmann, 1995) y biográfico-narrativo (Bolívar y Domingo, 2019). Lo anterior, con el fin de aprehender los sentidos construidos por los mismos actores en su cotidianeidad (Creswell, 2013). Desde esta óptica, resulta de suma relevancia la visión de las directoras y el análisis contextual que realizan en sus discursos.

Por otra parte, esta investigación se basó en un estudio instrumental de casos para abordar los cambios educativos en sus propios contextos (Stake, 1999). El estudio de caso supone un abordaje desde lo singular y los distintivos (Simons, 2011), situación que permitió ahondar en hechos específicos de interés para la investigación y otorgó la oportunidad de captar y reflejar los elementos que dan significado al objeto de estudio en cuestión.

Para acercarnos al objeto de estudio, se trabajó desde el método biográfico (Bolívar y Domingo, 2019), más específicamente desde la técnica de investigación llamada relato de vida (Bertaux, 2005), entendido como una expresión de una parte de la vida de las directoras en estudio, que requiere de un tiempo, secuencia y espacio definido para su unidad, además de una trama, una secuencia, un protagonista y una situación para configurarse como una narración biográfica (Bolívar y Domingo, 2019). Con esta técnica recogimos el lenguaje de las directoras, ya sea oral o escrito, y de esta forma se pudo conocer el interior de ellas, sus significados de vida.

Esta investigación contó con la participación de diez directoras de establecimientos educacionales de enseñanza básica de la Región Metropolitana de Chile. La selección de los casos fue de manera equitativa a partir del tipo de dependencia de los establecimientos educacionales (cinco municipalizados y cinco subvencionados particulares). 
Tabla 1

Características de las directoras participantes

\begin{tabular}{lcccc}
$\begin{array}{c}\text { Directoras } \\
\text { participantes }\end{array}$ & $\begin{array}{c}\text { Dependencia del } \\
\text { establecimiento }\end{array}$ & $\begin{array}{c}\text { Años de experiencia } \\
\text { como docente }\end{array}$ & $\begin{array}{c}\text { Años de experiencia } \\
\text { en cargos directivos }\end{array}$ & $\begin{array}{c}\text { Ańos de experiencia } \\
\text { como directora }\end{array}$ \\
\hline Directora No1 & Subvencionado particular & 16 años & 3 años & 2 años \\
\hline Directora No2 & Subvencionado particular & 5 años & 25 años & 2 años \\
\hline Directora No3 & Municipal & 21 años & 4 años & 6 años \\
\hline Directora No4 & Municipal & 10 años & 8 años & 7 años \\
\hline Directora No5 & Subvencionado particular & 15 años & 8 años & 2 años \\
\hline Directora No6 & Municipal & 25 años & 3 años & 4 años \\
\hline Directora No7 & Municipal & 18 años & 24 años & 10 años \\
\hline Directora No8 & Municipal & 14 años & 4 años & 10 años \\
\hline Directora No9 & Subvencionado particular & 15 años & 10 años & 7 años \\
\hline Directora No10 & Subvencionado particular & 12 años & 4 años & 2 años \\
\hline
\end{tabular}

Fuente: elaboración propia.

Por otra parte, y atendiendo a la naturaleza de esta investigación, se utilizaron dos instrumentos que permitieron indagar en el objeto de estudio: cuestionario autobiográfico y entrevistas biográficas semiestructuradas tres por cada directora en estudio-. Ambos instrumentos fueron validados por un grupo de expertos.

Tabla 2

Grupo de expertos
Profesión - rol
Género
Rango años de
experiencia
Experticia

\begin{tabular}{|c|c|c|c|}
\hline $\begin{array}{c}\text { Docente - director de } \\
\text { establecimiento educativo }\end{array}$ & Hombre & $20-30$ años & Experiencia en el cargo de dirección escolar. \\
\hline $\begin{array}{l}\text { Docente - directora de } \\
\text { establecimiento educativo }\end{array}$ & Mujer & 10 a 20 años & Experiencia en el cargo de dirección escolar. \\
\hline $\begin{array}{l}\text { Académico - investigador } \\
\text { Universidad Metropolitana }\end{array}$ & Hombre & 10 a 20 años & $\begin{array}{c}\text { Experticia en formación docente - directiva } \\
\text { universitaria. }\end{array}$ \\
\hline Investigador senior & Hombre & 20 a 30 años & $\begin{array}{c}\text { Experticia en formación de docente - directiva } \\
\text { universitaria y desde la política pública. }\end{array}$ \\
\hline $\begin{array}{l}\text { Académico - investigador } \\
\text { Universidad de Chile }\end{array}$ & Mujer & 10 a 20 años & $\begin{array}{c}\text { Experticia en investigación universitaria en } \\
\text { metodología biográfica narrativa. }\end{array}$ \\
\hline
\end{tabular}

Fuente: elaboración propia.

Esta validación tuvo como objetivo resguardar y asegurar la coherencia interna y externa de los instrumentos a partir de los criterios de pertinencia, claridad y relevancia. Este proceso contó con dos instancias; la primera supuso la realización de modificaciones estructurales, terminológicas y semánticas, que fueron aplicadas a los 
instrumentos, los que posteriormente fueron reenviados al grupo de expertos anteriormente mencionamos como validadores. En esta segunda fase se realizaron algunas modificaciones -principalmente referidas al aspecto, que fueron atendidas dando lugar a los instrumentos definitivos.

El análisis realizado estuvo asociado al enfoque biográfico narrativo presente en esta investigación, que buscó encontrar temas comunes en un conjunto de narraciones, posteriormente analizados mediante dos tipos de análisis; uno que surge desde el marco conceptual previo y otro que es construido de manera inductiva desde los mismos relatos (Bolívar, 2002).

Por ello, se optó por trabajar aquí desde la teoría fundamentada (Glaser \& Strauss, 1967), debido a la posibilidad que este método ofrece en la facilitación de los procesos de interpretación de experiencias a través del tiempo o transformaciones a través de etapas.

Una vez transcritos los relatos de vida, se trabajó con el software Atlas ti. 7, buscando fortalecer la coherencia y el rigor de los procedimientos analíticos. Para el análisis, se diseñó una matriz de dimensiones y categorías que contiene tanto los elementos teóricos como aquellos resultantes de la codificación abierta.

Tabla 3

Dimensiones y categorías de análisis de la investigación

$$
\begin{array}{cc}
\text { Conocimientos teóricos } & \text { Conocimientos prácticos o } \\
\text { o para la práctica. } & \text { en la práctica }
\end{array}
$$

- Ejercicio docente (primeras experiencias pedagógicas)

- Experiencias de consolidación docente

- Formación continua como directiva directora
- Ejercicio directivo

- Experiencias de consolidación directiva

$\begin{array}{cc}\text { Conocimientos } & * \text { Identidad y Trayectorias } \\ \text { experienciales reflexivos o } & \text { directivas } \\ \text { de la práctica } & \end{array}$

- Incidentes críticos de la gestión directiva

- Identidad directiva (autopercepción del rol)

- Caracterización de la gestión directiva

- Evaluación de la experiencia: obstaculizadores/ facilitadores

- Evaluación del cargo: obstaculizadores/ facilitadores de la gestión
- Experiencias de consolidación docente y directiva

- Elementos destacados de la experiencia docente y directiva

- Valoración experiencias docentes y directivas

Fuente: elaboración propia.

\section{Resultados}

\section{Construcción de conocimiento profesional}

En referencia a la construcción del conocimiento profesional, las directoras hacen continua mención a tres dimensiones específicas - tres saberes diferentes- que se articulan permitiendo que emerja el conocimiento necesario para el ejercicio continuo de su profesión. Dichas dimensiones son: conocimiento teórico (formal) para la práctica, conocimiento práctico (experiencia) en la práctica, y conocimiento experiencial reflexivo de la práctica (Porlán-Ariza et al., 1997; Shulman, 2005; Demuth, 2011; Simkins, 2005). 


\section{Conocimiento teórico o para la práctica}

Un primer elemento que resalta a la hora de pensar la construcción de conocimiento hace referencia a los saberes teóricos. (Porlán-Ariza, 1997). A este respecto, existe una gran valoración por parte de las entrevistadas con respecto al conocimiento teórico a la hora de pensar su practicas directivas. Principalmente por que ven en este la responsabilidad de mantener actualizado su conocimiento, cuestión que consideran vital para el ejercicio de su rol.

La formación continua es la que le va a ir dando cada vez más herramientas, las actualizaciones que una tiene que tener... (Directora No 7).

Según las entrevistadas, el conocimiento teórico tiene un importante rol que se expresa en relación con la puesta en acción del ejercicio directivo (Marcelo, 2009). Para ellas, el conocimiento teórico permite la construcción de un banco de herramientas profesionales que facilitan el acercamiento y desarrollo de la labor directiva. En esta línea, se observa un primer vínculo con el componente de interacción social que se encuentra en la base de la construcción del conocimiento, ya que a juicio de las entrevistadas el conocimiento teórico permite dar respuestas a interrogantes que van surgiendo desde el vínculo que existe con las comunidades pedagógicas, en la práctica misma (Tardif, 2004).

Entonces, qué pasa, yo creo que me apoyé mucho de la teoría del magíster, pero como estaba en la práctica... entonces yo creo que se me hizo más fácil por eso, lo estaba viviendo (Directora No 10).

Por otro lado, las entrevistadas ponen especial énfasis en el aporte que entregan a su labor los espacios de formación continua -asociados al aprendizaje de conocimientos teóricos-. Para ellas, además de entregarles conocimientos académicos, estos se constituyen en espacios de socialización entre pares directivos, que les permiten un ejercicio de retroalimentación a partir de las experiencias personales y grupales.

Una de las buenas instancias donde uno resolvía y encontraba soluciones a algunos problemas era cuando hice los cursos, el plan de formación de directores (...) Entonces, ese espacio de diálogo y de participación es muy enriquecedor, creo que uno debería tener esas instancias (Directora No 8).

En este sentido, los espacios de formación continua permiten además de la formación teórica la construcción de un conocimiento reflexivo colectivo (Schön, 1992) que surge de este vínculo entre pares. En esta línea, y a juicio de las entrevistadas, una de las principales virtudes que presentan estos cursos de formación continua es precisamente motivar el ejercicio reflexivo-crítico entre quienes participan de ellos, promoviendo la interacción y la producción de conocimiento profesional (Campos, 2016).

\section{Conocimiento práctico o en la práctica}

Las directoras le otorgan gran preponderancia al conocimiento práctico, ya que consideran que permite situar su liderazgo, poniendo en acción lo aprendido, tensionando constantemente sus pautas de acción (Porlán-Ariza et al., 1997), lo que implica necesariamente un proceso de revisión y continua actualización del conocimiento, posicionándose así como un dinamizador de la construcción de este mismo, relacionándolo con el saber hacer, en continuo movimiento y en continua acción de la práctica (Pérez Gómez, 1998).

La sala de clases, para mí eso ha sido muy formador. Lo que no quiere decir que la universidad haya sido menos, en las distintas instancias que estuve, pero todavía es más abstracta la figura en términos de formación profesional; creo que la concreción de eso, y la formación concreta, la he logrado en la práctica de lo que he hecho, y concretamente la práctica que he tenido en este colegio (Directora No 10). 
Para las directoras, es el vínculo continuo entre experiencia y teoría lo que les permite construir su conocimiento y las pautas de acción en el marco cotidiano de sus prácticas. Es más, pareciera ser imposible separar los componentes de ambos en el proceso de construcción de conocimiento (Marcelo, 2009).

La experiencia y la práctica por sí sola no tiene, no sirven de nada, requieren de lo teórico (Directora No 4).

Para las entrevistadas, la principal función que cumple el conocimiento práctico es que permite un espacio para el desarrollo de un conocimiento vivido, poner en acción los aprendizajes adquiridos (Tamir, 2005). Es decir, pone en tensión la posición que ocupan las directoras motivando un trabajo que busca abordar y solucionar las problemáticas que surgen en la cotidianeidad de las comunidades de aprendizaje y, a la vez, permite darle cuerpo a la teoría, que muchas veces se piensa de manera abstracta.

(...) Yo creo que la experiencia, el poder haber estado acompañando mucho a las directoras anteriores, porque en teoría puedes aprender muchas cosas, pero la práctica es la que te permite conocer y darle sentido a lo que estás haciendo (Directora No 2).

En este marco, no resulta extraño que las entrevistadas enlacen constantemente la teoría y la practica a la hora de reflexionar sobre sus conocimientos y sus experiencias, ya que, como señala Tadeu da Silva (2001), no se puede separar sujeto y objeto, el ser y el saber.

Creo que es la experiencia (es importante), pero acompańada por una cuota de conocimiento (Directora No 1).

Por otra parte, es posible identificar en el discurso de las directoras un claro énfasis referido a la experiencia y el rol que esta juega a la hora de visualizar los conocimientos que cada una tiene con respecto de su cargo y la forma en la que se vincula a diversos actores de las comunidades de aprendizaje (Bolívar, 2008; Fullan, 1999). En este sentido, la práctica les permite vivenciar las instituciones educativas como espacios dialécticos y dialógicos, cuestión que establece desafíos que deben ser enfrentados en su proceso de construcción de conocimiento.

Necesitas haber vivido la experiencia de ser profesor para poder después dirigir a un profesor po, orientarlo o... o ayudarlo, apoyarlo. Si no has sido profesor, cómo vas a sentir lo que él está sintiendo como profesor en el aula (Directora No 6).

\section{Conocimiento experiencial reflexivo o de la práctica}

En el discurso de las directoras entrevistadas se identifican diversos procesos de reflexión de sus experiencias, presentes en sus trayectorias, que han surgido a partir de la cotidianeidad de su labor. Dichos procesos de reflexión se posicionan como un elemento de aprendizaje relevante para entender no solo su rol al interior de las escuelas, sino que también las pautas de acción con la cuales enfrentan su cotidianeidad (Poggi, 2001; Campos, 2016).

Cuando me pasan situaciones fuertes de trabajo, yo no me estreso, o sea, yo creo que no me estreso, yo trato de ser lo más racional, lo más objetiva, juntar todos los elementos y decir ya, se resuelve así (Directora No 1 ).

En este sentido, se identifica en el proceso de construcción de conocimiento profesional un espacio reflexivo y crítico, resultado de la relación continua entre conocimiento teórico y práctico, y la reflexión de ambos en acción (Giddens, 1992; Campos, 2016). Dicho conocimiento contribuye a la formulación de la imagen situada de sus roles en los contextos específicos, como lo señala la siguiente directora. 
Pero la experiencia, o sea lo más teórico de verdad, siempre que lleves harto rodaje, tú puedes empezar a hacer las conexiones (reflexiones) entre ambos (Directora No 9).

Dicho proceso reflexivo se presenta en un orden diferente a los dos conocimientos previamente analizados, por que surge de la yuxtaposición de ambos en el marco de una práctica situada. Es la puesta en tensión e interacción de los saberes lo que promueve la emergencia de un componente reflexivo (Schön, 1992).

En este aspecto, se plantea que este conocimiento de carácter reflexivo requiere siempre de su vínculo con la experiencia, porque demanda de la puesta en acción para poder desarrollarse (Marcelo, 2009).

\section{Construcción de conocimiento profesional por dependencia de establecimiento educativo}

Hay un elemento que resalta como principal divergencia entre las directoras de ambas dependencias, asociado al valor que tienen el conocimiento teórico y práctico y la importancia del conocimiento experiencial en la construcción de la trayectoria profesional, habiendo distintas estrategias en la construcción de conocimiento profesional en ambos grupos de directoras.

\section{a. Estrategia de directoras de establecimientos municipales}

Estas directoras señalan que su proceso de construcción de conocimiento profesional se enmarca en una estrategia construida y enfocada en dar respuesta a las aptitudes y títulos requeridos para alcanzar un cargo directivo.

Yo siempre estuve muy interesada -en ser directora- y mi propósito era validarme (...), no exitista, porque no era para lucir, si no que para, para mí. Era como mi proyecto de vida, digamos (Directora No 7).

En este contexto, se observa una gran puesta en valor de los procesos de formación académicos y la experiencia exógena, en la medida en que entienden que estos les permiten conocer en profundidad el sistema escolar chileno, cuestión que resulta relevante para los concursos públicos para el cargo de director.

El tema de tener el magíster a ti te permite de alguna manera optar a algún cargo, al no tener una, era lo que yo pensaba en ese minuto, y eso no, porque yo podría haber optado antes al cargo de director, y no tenía magíster, entonces no tengo opciones (Directora No 8).

Otro elemento que destacan las entrevistadas es la búsqueda por avanzar en las escalas jerárquicas al interior de las escuelas. A su juicio, es de suma importancia poder acceder a cargos directivos antes de postular a un cargo de director, porque entienden que estos las preparan para el desafío que implica ser directora. Este elemento presente, en la forma en que las entrevistadas construyen su conocimiento, dice relación con una promoción vertical (UNESCO, 2007) en el sistema educativo.

(...) La directora debe tener experiencia directiva antes de asumir la dirección. Tiene que tener la experiencia de haber sido o jefe de UTP o inspector general, o subdirector. Yo creo que debiera pasar por alguno de esos... (Directora No 7).

De esta forma, lo relevante en esta promoción es avanzar en cargos que implican nuevas y más grandes responsabilidades. Esto dice relación con los procesos de selección al cargo de director del sistema público, ya que uno de los criterios de evaluación a considerar al momento de la selección es la experiencia anterior y su desempeño en ella (Ley No 19.070, 1997). En este sentido, podemos observar una estrategia situada en sus trayectorias que se adapta a los requerimientos del sistema educativo, a las exigencias de las políticas públicas actuales - principalmente concursabilidad y evaluación del cargo: Ley de Equidad y Calidad de la Educación (No 20.501, 2011) o la ley que establece la concursabilidad de los cargos de directores de establecimientos 
educacionales municipales (Ley No 20.006, 2005) - las cuales permiten estandarizar criterios de selección. A raíz de esto, es posible identificar a lo largo de su trayectoria una estrategia en la que se busca abarcar todos los requerimientos para acceder al cargo de dirección.

\section{b. Estrategias de directoras de establecimientos particulares subvencionados}

Por otro lado, el camino que lleva a las directoras de colegios subvencionados a alcanzar posiciones directivas se caracteriza por otorgar gran importancia a la trayectoria y confianzas al interior de los establecimientos educativos.

En el caso de las escuelas particular subvencionadas, el proceso de selección de las directoras se basa en confianzas construidas en el trabajo cotidiano y sostenido en las instituciones. De tal forma, más que requisitos específicos, lo relevante es cuánto y cómo se enmarcan sus perfiles en el marco de la visión y la misión de los colegios. Es tan importante este componente que a veces deja en segundo plano requisitos técnicos o curriculares (posgrados).

Me dice 'sabes que la comunidad religiosa cree que tú debes participar', entonces yo le dije 'yo no lo había contemplado, no tengo ni siquiera mi currículum actualizado', 'pero voy a participar', 'pero ustedes tienen que obviar esos dos requisitos, yo participo', me dijeron 'sí, mira, ya llevamos siete entrevistas de las 12 personas, y la verdad es que yo creo que ninguno representa lo que tu podrías hacer en el cargo' (Directora No 10).

En este sentido, se observa que la estrategia utilizada por este grupo de directoras presenta rasgos más flexibles; su proceso de construcción de conocimiento profesional, principalmente referido a lo académico, es algo que surge de manera más espontánea y con un gran apoyo, lo que les permite construir su currículum con más libertad.

No, el colegio se preocupa mucho por eso (formación), algunos fueron por el colegio, que son, cuando se hace fin de año, uno postula, le dice 'hermana, mire está la posibilidad, hay recursos, o miti-mota'; algunos fueron gestionados por el colegio, y otros fueron gestionados por mí (Directora No 1).

Esta manera de abordar la construcción del conocimiento profesional en el caso de las directoras de colegios subvencionados, y junto con sus contextos específicos, se relaciona con una promoción horizontal (UNESCO, 2007).
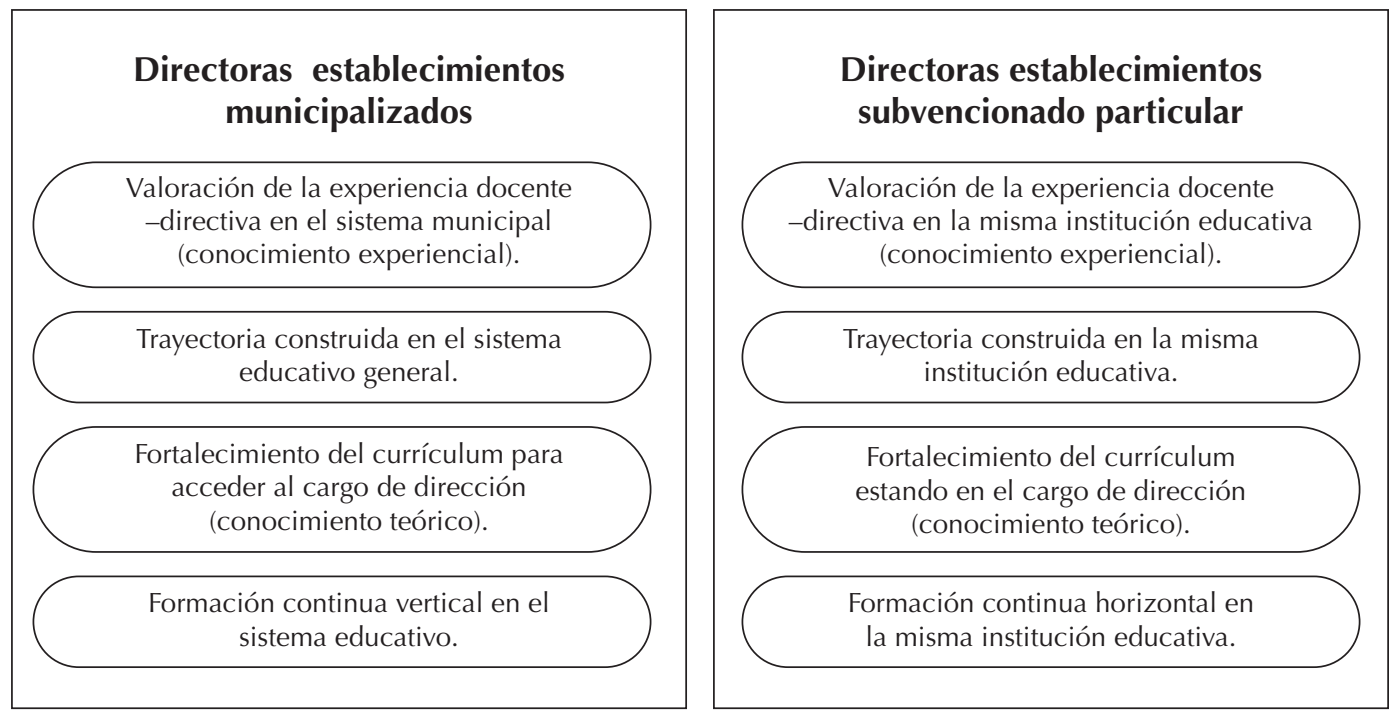

Figura 2. Sintesis de construcción del conocimiento profesional de directoras, por dependencia Fuente: elaboración propia.

1. Expresión chilena que quiere decir "mitad y mitad" o " $50 \%$ y 50\%". 


\section{Identidades de las mujeres directoras}

En relación con esta dimensión, encontramos elementos de identidad de las directoras que se presentan como comunes entre todas, principalmente los asociados a la dimensión más personal (Sammons, 2007), y otros que difieren. Con respecto a los primeros, las directoras señalan que el hecho de ser mujeres $-y$ todo lo que ello conlleva- es un elemento relevante a la hora de pensar su identidad.

Las directoras van forjando su identidad en una continua relación entre su imagen de sí mismas y la imagen que los demás tienen de ellas (Dubar, 2002). En este sentido, no resulta extrańo que esta dificultad para validarse frente a los demás vaya mermando su confianza.

Porque el hombre llega validado porque es hombre en esta cultura machista, y la mujer se tiene que ganar esa validación. Y eso es verdad, es triste, pero es verdad. Yo recuerdo que llegó, en un lugar donde yo trabajaba, llegó un director y 'llegó director, qué bueno que es hombre'. Se había ido una directora hacía poco, no les había gustado, ya tenía que salir... 'Y qué bueno que llegó, ahora las cosas van a ser diferentes, porque es distinto estar con...' Yo decía: “Dios mío, qué machismo más exacerbado (Directora No 4).

De acuerdo con lo anterior, se evidencian los conflictos que se generan al interior de esta directora, donde el feminismo se yergue como un medio de transformación de los espacios patriarcales, heteronormados y masculinizados (Barberá et al., 2011; Armstrong \& Mitchell, 2017).

Por otra parte, el rol directivo está caracterizado típicamente por lo masculinizado (Barberá et al., 2011), por la posición de poder que se configura en este espacio en el que el trabajo es lo primero, cuestión que resulta difícil de compatibilizar con aspectos del cuidado del hogar y de los hijos (Cabrera, 2005; Coronel et al., 2012; Cubillo \& Brown, 2003; Gómez y Jiménez, 2019).

Frente a esto, las directoras señalan la necesidad de construir una imagen que refuerce su capacidad para cumplir en todas las esferas en las que participan, incluso soportando actos que implican vulnerar sus propios derechos, hacer una maternidad machista (Smithson \& Stokoe, 2005).

Antes de tener a mi último hijo, yo ese día vine a trabajar, vine a una reunión porque había que preparar la licenciatura y la hermana me llamó, yo vine y ese mismo día tuve mi guagua ${ }^{2}$ (Directora № 2).

Lo anterior deja nuevamente en evidencia las formas de dominación patriarcales que se despliegan en el espacio escolar, las que develan la necesidad de impulsar prácticas centradas en pedagogías feministas (Troncoso et al., 2019). Además, se observa en el discurso de las entrevistadas la tendencia a construir su trayectoria personal y profesional asociada a la imagen de una gerente ideal (Moorosi, 2007) que logre responder a todas los imposiciones que histórica y socialmente se han construido en este espacio masculinizado.

Yo me caso en la época que estaba en el Pehuén, claro, y nace Fernanda, mi hija... mi segunda hija, pero ahí pasó un tema, como yo estaba a cargo de todo no pude dejar de trabajar, y los sostenedores me pidieron, la dueña, que ellos me pagaban doble sueldo, pero que yo no me fuera. Entonces, mi hija nació un día jueves y yo trabajé un día miércoles hasta las 6:00 de la tarde, y volví a trabajar un día martes de la siguiente semana con la niña” (Directora № 6).

En este sentido, este rol de gerente ideal es una figura que busca cumplir con un compromiso desmedido con las tareas asignadas al rol, ya que deben rendir al máximo tanto en la esfera profesional como en la personal, resultando en identidades de mujeres que por aceptar el reconocimiento de otros transan su rol de madres. Esto se debe mayormente a que la característica propia de la masculinidad hegemónica en el ejercicio de la dirección escolar atenta contra el desarrollo de la práctica femenina en los cargos de liderazgo (Blackmore, 2017).

2. Chilenismo para "bebé". 


\section{Identidades directivas en contextos (por dependencia)}

A este respecto, resulta posible señalar que se observan distinciones en las identidades construidas por las directoras, principalmente referidas a la dependencia de sus establecimientos educacionales. Dichos procesos dicen relación con la forma en que estas instituciones entienden el rol directivo, cuestión que, como vimos, también actúa fuertemente en el proceso de construcción de conocimiento previamente discutido.

En el caso de las instituciones municipalizadas, es posible observar la existencia de un proceso de construcción de identidad estándar vinculado a la homogeneidad que requiere el sistema público a la hora de pensar el cargo directivo. En este sentido, y siguiendo los lineamientos de Sammons (2007), es posible observar en estas identidades construidas una mayor relevancia de la dimensión referida a la tradición de la enseñanza y de las políticas educativas, a la vez que se observa la importancia que le otorgan a ciertos criterios formalizados y estandarizados en el marco de sus propias identidades, una trayectoria más exógena.

Yo postulé al concurso para la escuela rural, porque yo cuando hice el magíster dije 'ya, tengo que tener otra experiencia educativa', entonces empecé a buscar concurso que hubiera en colegios rurales. Y postulé a la comuna de María Pinto. Y entonces ahí me fui a trabajar a la escuela rural de Santa Emilia, que en ese momento estaba entre las 10 peores de Chile. Entonces para mí era súper práctico (Directora No 4).

En el caso de las instituciones particulares subvencionadas, es posible observar un proceso de construcción de la identidad en el cual se le otorga el principal valor a la dimensión referida a los criterios locales y relacionados con la escuela y la institución o las instituciones por las que se ha transitado (Sammons, 2007). En este sentido, se observa una identidad que refiere constantemente a la trayectoria endógena de las entrevistadas en cada institución, la que les permite orientar sus identidades enmarcadas en la misión/visión particular de la comunidad de aprendizaje, más que lo estandarizado en el sistema educativo.

Yo a usted la visualizo, yo sé cómo ha sido su experiencia laboral, y yo necesito que usted lo sea (...) Ella me lo dijo, en estos años ella me había observado, que tenía primero el carácter de la madre fundadora, entonces yo le dije '¿cuál es ese?', entonces me dijo 'tu trabajas mucho en beneficio de los niños, por tu rol de psicopedagoga, siempre estás apoyando, siempre estás ahí, y eso es lo que yo quiero', y yo bien agradecida (Directora No 1).

De esta forma, es posible observar en el proceso de construcción de identidades directivas una fuerte influencia de la dependencia de los establecimientos en los que se desarrollan las directoras.

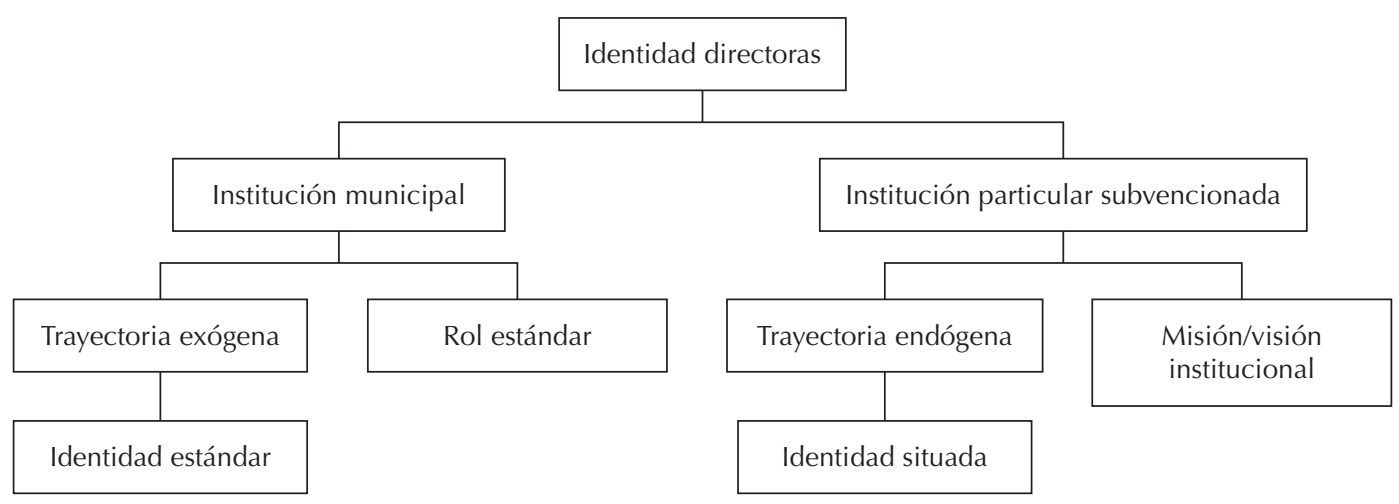

Figura 3. Esquema comparativo de las identidades en contexto (dependencia). Fuente: elaboración propia. 


\section{Conclusiones}

La construcción de conocimiento profesional e identidad de directoras de establecimientos de enseñanza básica chilena es un proceso situado, de interacción social, mediante el cual se conjugan saberes teóricos, prácticos y reflexivos, todos en la base de la experiencia del sujeto, ya sea individual o colectiva. Es una construcción que no puede separarse de la experiencia de vida personal, que en este caso condiciona el tipo de directoras: capaces de transar su vida personal-familiar por lograr sus objetivos profesionales. Ser mujeres en un espacio escolar altamente masculinizado y patriarcal (Barberá et al., 2011; Coleman, 2007, Blackmore, 2017) ha condicionado sus trayectorias e identidades profesionales, convirtiéndolas en mujeres con un alto grado de compromiso por cumplir con las exigencias históricamente impuestas, así como en directoras con una gama de conocimientos profesionales, fruto de las experiencias de la práctica y del aprendizaje en formación continua.

Si bien los elementos aquí discutidos son resultado del trabajo sistemático a partir del discurso de diez directoras chilenas, es un estudio de caso que no ha pretendido generalizar, sino que más bien busca entregar reflexiones y orientaciones que permitan aportar a la discusión de las políticas públicas, y ser un punto inicial para -a futurocontinuar profundizando en esta temática.

En primer lugar, para las directoras de este estudio, el conocimiento teórico por sí solo no tiene valor: debe estar vinculado con el conocimiento práctico y, a su vez, contextualizado en las prácticas del quehacer directivo. A partir de esta relación situada entre los saberes teóricos y prácticos surge un tercer componente relevante, según las directoras de este estudio, para la construcción del conocimiento: el conocimiento reflexivo experiencial. Este se encuentra en un orden diferente a los previamente mencionados, ya que surge desde la yuxtaposición de ambos en una práctica situada. De esta forma, se observa en el discurso de las entrevistadas una referencia continua a este saber que surge desde la cotidianeidad de la práctica educativa: es en las situaciones con posibilidad de mejora que las directoras ponen en acción/revisión sus modos de actuar, construyendo así nuevas pautas de acción.

Por lo anterior, es importante considerar que, a la hora de pensar en la concursabilidad y la evaluación del cargo de directoras, se debe considerar el conocimiento experiencial como un componente clave; sin embargo, no debe entenderse como un conocimiento de experiencia que solo considere el número o tipo de programas realizados, sino que incluya también la experiencia como la relación entre los saberes teóricos y prácticos que permite generar reflexiones y buscar soluciones a situaciones o incidentes cotidianos de la práctica directiva a través de un caso.

De igual modo, para estas directoras las experiencias docentes y directivas previas a asumir el cargo de dirección (en diferentes cargos de gestión) son un factor determinante y, por ende, muy valorado a la hora de concursar al cargo, ya que para ellas las prácticas docentes y de gestión permiten tener un conocimiento profesional más preparado a la hora de asumir la responsabilidad.

En segundo lugar, se concluye que, respecto de la identidad femenina de estas directoras, es evidente que el rol de mujeres ha condicionado sus trayectorias profesionales, por lo que no se puede soslayar que la identidad de estas 10 directoras se configura en torno a los prejuicios históricamente construidos sobre el rol de las mujeres, que las limitan y al mismo tiempo obstaculizan el desarrollo de un liderazgo femenino.

Por otra parte, se señala que desarrollar trayectorias profesionales en el mismo espacio educativo permite construir identidades directivas que se configuran con las características y cultura escolar de la institución, lo que genera una mejor interacción con los actores de la comunidad educativa, lo que queda en evidencia en las entrevistas de este estudio. Este tipo de identidad endógena permite mejores compromisos con la institución, mayor sentido de pertenencia y más vinculación con la misión y visión del establecimiento educativo, logrando un ejercicio comprensivo más eficiente y profundo, diferente a lo que ocurre con las directoras de establecimientos educacionales municipalizados, que construyen una identidad exógena. 
Por lo anterior, se sugiere que la concursabilidad del cargo de director/a, debiese estar mucho más anclada a la experiencia del director/a en la institución en la que concursa, ya que se valoran los conocimientos experienciales y prácticos adquiridos en el mismo espacio educativo como un elemento destacado para un mejor ejercicio directivo.

Sin duda, en lo que respecta a identidad y trayectorias femeninas, es menester reflexionar sobre las políticas de género en el espacio escolar y, sobre todo, en las de cargos directivos, debido a que el número de mujeres en estos es cada día más alto; así, repensar la gestión y el liderazgo desde la óptica femenina es una temática a resolver, ya que en la actualidad el marco de actuación de las directoras sigue estando ligado a una estructura masculinizada. Urge superar la lógica heteronormativa, desigual y discriminatoria de lo patriarcal, y transitar hacia prácticas pedagógicas femeninas, situadas en la justicia social, lo colectivo y en la correspondencia de roles y funciones.

Finalmente, pensar una carrera directiva para el país debiese tener presente los elementos anteriormente mencionados, ya que un ejercicio directivo eficiente que permita implementar un liderazgo para la mejora de la institución requiere considerar el contexto y las características de la institución educativa, así como la del o la directiva que asuma.

El artículo original fue recibido el 5 de enero de 2020

El artículo revisado fue recibido el 3 de noviembre de 2020

El artículo fue aceptado el 7 de enero de 2021

\section{Referencias}

Armstrong, D. \& Mitchell, C. (2017). Shifting Identities. Negotiating Intersections of Race and Gender in Canadian Administrative Contexts. Educational Management Administration \& Leadership, 45(5), 825-841. https://doi.org/10.1177/1741143217712721

ATLAS.ti (Versión 7) [Software de computación]. Berlin: Scientific Software Development GmbH.

Barber, M. y Mourshed, M. (2008). Cómo hicieron los sistemas educativos con mejor desempeño del mundo para alcanzar sus objetivos. Santiago, Chile: PREAL.

Barberá, E., Ramos, A., y Candela, C. (2011) Laberinto de cristal en el liderazgo de las mujeres. Psicothema, 23(2), 173-179. Recuperado de http://www.psicothema.com/psicothema.asp?id=3867

Berger, P. y Luckmann, T. (1995). La construcción social de la realidad. Buenos Aires, Argentina: Amorrortu.

Bertaux, D. (2005). Los relatos de Vida. Barcelona, España: Ediciones Bellaterra.

Blackmore, J. (2017). 'In the shadow of men': the historical construction of administration as a 'masculinist' enterprise. En J. Blackmore \& J. Kenway (Eds.), Gender Matters in Educational Administration and Policy (pp. 27-49). Londres, Reino Unido: Routledge.

Bolívar, A. (2002). “¿De nobis ipsis silemus?”: Epistemología de la investigación biográfico-narrativa en educación. Revista Electrónica de Investigación Educativa, 4(1), 2-26. Recuperado de https://redie.uabc.mx/redie/article/view/49

Bolívar, A. (2007). La formación inicial del profesorado de secundaria y su identidad profesional. Estudios Sobre Educación, 12, 13-30. Recuperado de https://revistas.unav.edu/index.php/estudios-sobre-educacion/article/view/24326

Bolívar, A. (2008). Avances en la gestión e innovación de los centros. En A. Villa (Ed.), Innovación y cambio en las organizaciones educativas (pp. 291-317). Bilbao, España: ICE de la Universidad de Deusto.

Bolívar, A. (2019). Una dirección escolar con capacidad de liderazgo pedagógico. Madrid, España: Arco Libro- La Muralla. Bolívar, A. y Domingo, J. (2019). La investigación (auto)biográfica en educación. Barcelona, España: Octaedro. 
Bolívar, A. y Ritacco, M. (2019). La identidad profesional de la dirección escolar. Una nueva línea de investigación sobre liderazgo escolar exitoso. En M. A. Díaz \& A. Veloso (Coords), Modelos de investigación en Liderazgo Educativo: una revisión internacional (pp. 147-159). Ciudad de México, México: Universidad Nacional Autónoma de México.

Bustos, C., Oros, S., Álvarez, D., y Rebolledo, B. (2015). Presencia del enfoque de género en la gestión escolar en Chile. Temas de Educación, 21(1), 97-115. Recuperado de https://revistas.userena.cl/index.php/teduacion/article/view/655

Cariño, C., Cumes, A., Curiel, O., Garzón, M. T., Mendoza, B., Ochoa, K., y Londoño. (2017). Pensar, hacer y sentir pedagogías feministas descoloniales: diálogos y puntadas. En C. Walsh (Ed.), Pedagogías decoloniales. Pedagogías insurgentes de resistir, (re) existir) y (re) vivir. Tomo II. (pp. 509- 536). Quito, Ecuador: Abya Yala.

Cabrera, L. (2005). Mujer, trabajo y sociedad (1839-1983). Madrid, España: Fundación F. Largo Caballero.

Campos, J. (2016). Conocimiento profesional de los profesores: formación de profesores en dos agrupamientos de escuelas. Revista Electrónica Interuniversitaria De Formación Del Profesorado, 19(2), 273-287. Recuperado de https://revistas.um.es/reifop/article/view/255681

Carver, C. (2016). Transforming identities. The transition from teacher to leader during teacher leader preparation, Journal of Research on Leadership Education, 11(2), 158-180. https://doi.org/10.1177/1942775116658635

Coleman, M. (2007). Gender and educational leadership in England: A comparison of secondary headteachers' views over time. School Leadership and Management, 27(4), 383-399. https://doi.org/10.1080/13632430701562991

Contreras, J. y Pérez de Lara, N. (2010). La experiencia y la investigación educativa. En J. Contreras y N. Pérez de Lara (Comps.), Investigar la experiencia educativa (pp. 21-86). Madrid, España: Morata.

Coronel, J., Llamas, M., Macías, C., \& Moreno, E. (2012). Overcoming Obstacles and Problems: a Multicase Study of Women Principals, Leadership Policies and Management for School. Revista de Educación, 357, 537-559. https://doi.org/10.4438/1988-592X-RE-2011-357-072

Creswell, J. (2013). Investigación Cualitativa y Diseño Investigativo. Selección entre cinco tradiciones. Los Ángeles, CA: Sage.

Crow, G. \& Møller, J. (2017). Professional identities of school leaders across international contexts: An introduction and rationale. Educational Management Administration and Leadership, 45(5), 749-758. https://doi.org/10.1177/1741143217714485

Cubillo, L. \& Brown, M. (2003). Women into educational leadership and management: international differences?. Journal of Educational Administration, 41(3), 278-291. https://doi.org/10.1108/09578230310474421

Demuth, P. (2011). Conocimiento profesional docente: conocimiento académico, saber experiencial, rutinas y saber tácito. Revista del Instituto de Investigaciones en Educación Facultad de Humanidades, (2), 29-46. https://doi.org/10.30972/riie.023699

Dubar, C. (2002). La crisis de identidades. La interpretación de una mutación. Barcelona, España: Edicions Bellaterra.

Dubet, F. (2011). La experiencia sociológica. Barcelona, España: Ediciones Gedisa.

Fullan, A. (1999). Las fuerzas del cambio: Explorando las profundidades de la reforma educativa. Madrid, España: Akal.

Giddens, A. (1992). La constitución de la sociedad: bases para la teoría de la estructuración. Buenos Aires, Argentina: Amorrortu.

Glaser, B. \& Strauss, A. (1967). Discovery of grounded theory. Chicago, IL: Aldine

Gómez, V. y Jiménez, A. (2019). Género y Trabajo: Hacia una agenda nacional de equilibrio trabajo familia en Chile. Revista Convergencia, 26(79), 1-24. https://doi.org/10.29101/crcs.v0i79.10911

Hargreaves, A. y Fullan, M. (2014). Capital Profesional. Madrid, España: Morata.

Heller, A. (1977). Sociología de la vida cotidiana. Barcelona, España: Península.

Jones, D. (2017). Constructing Identities: Female Head Teachers' Perceptions and Experiences in the Primary Sector. EducationalManagementAdministration \& Leadership, 45(6),907-928. https://doi.org/10.1177/1741143216653973

Lave, J. \& Wenger, E. (1991). Situated learning: Legitimate peripheral participation. Cambridge, Reino Unido: Cambridge University Press. Recuperado de http://wendynorris.com/wp-content/uploads/2018/08/LaveWenger-1991-Legitimate-Peripheral-Participation.pdf 
Leithwood, K., Day, C., Sammons, P., Harris, A., \& Hopkins, D. (2006). Successful School Leadership. What it is and how it influences Pupil learning. Londres, Reino Unido: University of Nottingham.

Leithwood, K. (2009). ¿Cómo liderar nuestras escuelas? Aportes desde la investigación. Santiago, Chile: Fundación Chile.

Ley No 19.070. Diario Oficial de la República de Chile, Santiago, Chile, 22 de Enero de 1997. Recuperado de https://www.bcn.cl/leychile/navegar?idNorma=60439

Louis, K. S., Leithwood, K., Wahlstrom, K. L., \& Anderson, S. E. (2010). Learning from leadership: Investigating the links to improved Student Learning. Recuperado de https://www.wallacefoundation.org/knowledge-center/ pages/investigating-the-links-to-improved-student-learning.aspx

Marcelo, C. (2009). Desarrollo Profesional Docente. Madrid, España: Narcea.

Marcelo, C. (2010). La identidad docente: constantes y desafíos. Revista Interamericana de Investigación, Educación y Pedagogía, 3(1), 15-42. https://doi.org/10.15332/s1657-107X.2010.0001.01

Maureira, O. y Garay, S. (2019). Liderazgo distribuido en escuelas primarias efectivas. En M.A. Díaz \& A. Veloso (Coords), Modelos de investigación en Liderazgo Educativo: una revisión internacional (pp. 147-159). Ciudad de México, México: Universidad Nacional Autónoma de México.

MINEDUC. (2016). Guía Metodológica para la elaboración y seguimiento de los convenios de desempeño de directores escolares. Santiago, Chile: Mineduc.

MINEDUC. (2019). Bases de datos del Centro de estudios. Recuperado de http://datos.mineduc.cl/dashboards/20031/ descarga-bases-de-datos-de-cargos-docentes/

Morgade, G. (2005). El encuentro entre docentes y practicantes como espacio privilegiado de construcción de conocimiento. En Actas del Primer Seminario Taller Internacional de Educación (pp.75-78). Buenos Aires, Argentina: Secretaría de Educación.

Moorosi, P. (2007). Creating linkages between private and public: challenges facing women principals in South Africa. South African Journal of Education, 27(3), 507-521.

Muñoz, G. y Marfán, J. (2011). Competencias y formación para un liderazgo escolar efectivo en Chile. Pensamiento educativo. Revista de Investigación Educacional Latinoamericana, 48(1), 63-81. Recuperado de http://pensamientoeducativo.uc.cl/index.php/pel/article/view/25645

Pech, J. y Morejón, P. (2018). La complejidad del conocimiento profesional docente y la formación del conocimiento práctico del profesorado. Revista Actualidades investigativas en educación, 18(2), 1-24. https://doi.org/10.15517/aie.v18i2.33129

Pérez Gómez, A. (1988). El pensamiento práctico del profesor/a. Implicaciones en la formación del profesorado. En V. Aurelio (Coord.), Perspectivas y problemas de la función docente (pp. 128-148). Madrid, España: Narcea.

Poggi, M. (2001). La formación de directivos de instituciones educativas Algunos aportes para el diseño. Buenos Aires, España: IIPE/UNESCO.

Porlán-Ariza, R., Rivero, A., y Martín del Pozo, R. (1997). Conocimiento profesional y epistemología de los profesores I: teoría, métodos e instrumentos. Enseñanza de Las Ciencias: Revista de Investigación y Experiencias Didácticas, 15(2), 155-172. Recuperado de http://hdl.handle.net/11441/25530

Ricoeur, P. (1996). Sí mismo como otro .Madrid, España: Siglo XXI.

Ritacco, M. y Bolívar, A. (2018). Identidad profesional y dirección escolar en Espańa: La mirada de los otros. Revista Brasileira de Educação, 23, 1-24. https://doi.org/10.1590/s1413-24782018230083

Sammons, P., Day, C., Kington, A., Gu, Q., Stobart, G., \& Smees, R. (2007). Exploring variations in teachers' work, lives and their effects on pupils: key findings and implications from a longitudinal mixed- method study. British Educational Research Journal, 33(5), 681-701. https://doi.org/10.1080/01411920701582264

Simons, H. (2011). El estudio de caso: teoría y práctica. Madrid, España : Morata.

Schön, D. (1992). La formación de los profesionales reflexivos. Barcelona, España: Paidós.

Shulman, L. (2005). Conocimiento y Enseńanza: Fundamentos de la Nueva Reforma. Profesorado. Revista de Currículum y Formación Del Profesorado, 9(2), 1-31.

Simkins, T. (2005). Leadership in education: "What works" or "what makes sense? Education Management Administration and Leadership, 33(1), 9-26. https://doi.org/10.1177/1741143205048168 
Smithson, J. \& Stokoe E., (2005). Discourses of Work-Life Balance: Negotiating 'Genderblind' Terms in Organizations. Gender, Work and Organization, 12(2), 147 - 168. https://doi.org/10.1111/j.1468-0432.2005.00267.x

Stake, R. (1999). Investigación con Estudio de Casos. Madrid, España: Ediciones Morata.

Tadeu da Silva, T. (2001). Espacios de identidad. Nuevas visiones sobre el currículum. Barcelona, España: Octaedro.

Tamir, P. (2005). Conocimiento profesional y personal de los profesores y de los formadores de profesores. Revista del Curriculum y formación del profesorado, 9(2), 263-268.

Tardif, M. (2004). Los saberes docentes y su desarrollo profesional. Madrid, Espańa: Narcea.

Tardif. M. (2013). El oficio docente en la actualidad. Perspectivas internacionales y desafíos a futuro. En M. Poggi (Ed.), Politicas docentes. Formación, trabajo y desarrollo profesional (pp. 19-44). Buenos Aires, Argentina: IIPE/Unesco.

Troncoso, L., Follegati, L., y Stutzin, V. (2019). Mas allá de una educación no sexista: aportes de pedagogías feministas interseccionales. Revista de Investigación Educacional Latinoamericana, 56(1), 1-15. Recuperado de http://pensamientoeducativo.uc.cl/index.php/pel/article/view/23411

UNESCO. (2007). Evaluación del Desempeño y Carrera Profesional Docente. Una Panorámica de América y Europa. Santiago, Chile: Andros impresores.

Uribe, M. \& Celis M. (2012) Desarrollo de capacidades para un liderazgo escolar efectivo: del levantamiento de competencias al reconocimiento de buenas prácticas. En J. Weinstein y G. Muñoz (Eds.), ¿Qué sabemos de los Directores en Chile? (pp. 111-134). Santiago, Chile: Salesianos Impresiones S.A.

Vaillant, D. (2008). La identidad docente. Importancia del profesorado. Revista Investigaciones en Educación, 8(1), 15-15. Recuperado de http://revistas.ufro.cl/ojs/index.php/educacion/article/view/942

Vaillant, D. (2019). Formación inicial del profesorado de educación secundaria en América Latina- dilemas y desafíos. Profesorado. Revista de Currículum y Formación de Profesorado, 23(3), 35-52. https://doi.org/10.30827/profesorado.v23i3.9516

Vanegas, O. \& Fuentealba, A. (2019). Identidad profesional docente, reflexión y práctica pedagógica: Consideraciones claves para la formación de profesores. Perspectiva Educacional, 58(1), 115-138. https://doi.org/10.4151/07189729-vol.58-iss.1-art.780

Weinstein, J. y Muñoz, G. (Eds.). (2012). ¿Qué sabemos de los Directores en Chile? Santiago, Chile: Salesianos Impresiones S.A.

Wenger, E. (2001). Comunidades de práctica. Aprendizaje, significado e identidad. Barcelona, España: Paidós. 